\title{
MHD Flow of an Incompressible Viscous Fluid through Convergent or Divergent Channels in Presence of a High Magnetic Field
}

\author{
Reza Hosseini, Sadegh Poozesh, and Saeed Dinarvand \\ Mechanical Engineering Department, Amirkabir University of Technology, \\ 424 Hafez Avenue, Tehran, Iran
}

Correspondence should be addressed to Reza Hosseini, hoseinir@aut.ac.ir

Received 20 September 2011; Accepted 7 November 2011

Academic Editor: C. Conca

Copyright (c) 2012 Reza Hosseini et al. This is an open access article distributed under the Creative Commons Attribution License, which permits unrestricted use, distribution, and reproduction in any medium, provided the original work is properly cited.

\begin{abstract}
The flow of an incompressible electrically conducting viscous fluid in convergent or divergent channels under the influence of an externally applied homogeneous magnetic field is studied both analytically and numerically. Navier-Stokes equations of fluid mechanics and Maxwell's electromagnetism equations are reduced into highly non-linear ordinary differential equation. The resulting non-linear equation has been solved analytically using a very efficient technique, namely, differential transform method (DTM). The DTM solution is compared with the results obtained by a numerical method (shooting method, coupled with fourth-order Runge-Kutta scheme). The plots have revealed the physical characteristics of flow by changing angles of the channel, Hartmann and Reynolds numbers.
\end{abstract}

\section{Introduction}

The incompressible viscous fluid flow through convergent or divergent channels is one of the most applicable cases in many applications such as aerospace, chemical, civil, environmental, mechanical, and biomechanical engineering as well as in understanding rivers and canals. Jeffery [1] and Hamel [2] have carried out the mathematical formulations of this problem in 1915 and 1916, respectively. If we simplify Navier-Stokes equations in the particular case of two-dimensional flow through a channel with inclined walls, finally we can reach JefferyHamel problem [3-6]. Jeffery-Hamel flows have been extensively studied by several authors and discussed in many textbooks, for example, [7-11], and so forth.

The study of electrically conducting viscous fluid that flows through convergent or divergent channels under the influence of an external magnetic field not only is fascinating 
theoretically but also finds applications in mathematical modeling of several industrial and biological systems. A possible practical application of the theory we envisage is in the field of industrial metal casting, the control of molten metal flows. Another area in which the theoretical study may be of interest is in the motion of liquid metals or alloys in the cooling systems of advanced nuclear reactors [12]. Clearly, the motion in the region with intersecting walls may represent a local transition between two parallel channels with different cross-sections, a widening or a contraction of the flow. The first recorded use of the word magnetohydrodynamics (MHD) is by Bansal [13]. The theory of MHD is inducing current in a moving conductive fluid in the presence of magnetic field which creates force on electrons of the conductive fluid and also changes the magnetic field itself. A survey of magnetohydrodynamics studies in the mentioned technological field can be found in [14]. The problem is basically an extension of classical Jeffery-Hamel flows of ordinary fluid mechanics to MHD. In the MHD solution an external magnetic field acts as a control parameter for both convergent and divergent channel flows. Here, beside the flow Reynolds number and the channel angular widths, at least an additional dimensionless parameter appears, namely, the Hartman number. Hence, a much larger variety of solutions than in the classical problem are expected.

Zhou was the first one who introduced DTM as an efficient method to apply for electrical circuits in his paper entitled "Differential transformation and its application for electrical circuits," [15]. It was used to solve both linear and nonlinear initial value problems in electric circuit analysis. The differential transform method (DTM) is an analytical method for solving ordinary differential equations, partial differential and integral equations. The method provides us with easily computable components and the solution is obtained in terms of convergent series. The main advantages of this method, compared to other analytic methods are controllable accuracy, and high efficiency, which is exhibited by the rapid convergence of the solution. The DTM gives exact values of the $n$th derivative of an analytic function at a point in terms of known and unknown boundary conditions. This method constructs, for differential equations, an analytical solution in the form of a polynomial. It is different from the traditional high-order Taylor series method, which requires symbolic computations of the necessary derivatives of the data functions. The disadvantage of Taylor series method is that this method computationally takes long time for large orders. The DTM is an iterative procedure for obtaining analytic Taylor series solutions of differential equations in a fast manner. This methodology introduces a promising approach for many applications in various domains of nonlinear problems. Various applications of DTM can be found in [1522].

The aim of the present work is to investigate the MHD flow through convergent or divergent channels in presence of a high magnetic field, by the differential transform method (DTM). The governing highly nonlinear equation of this problem is also solved numerically by shooting method, coupled with fourth-order Runge-Kutta scheme.

\section{Problem Statement and Mathematical Formulation}

Consider a system of cylindrical polar coordinates $(r, \theta, z)$, where the steady two-dimensional flow of an incompressible conducting viscous fluid from a source or sink at channel walls lie in planes and intersect in $z$-axis. The schematic diagram of problem is illustrated in Figure 1. We assumed that $u_{\theta}=0$; it means that there are no changes with respect to $z$ direction; thus the motion is purely in radial direction and merely depends on $r$ and $\theta[3,23]$, 


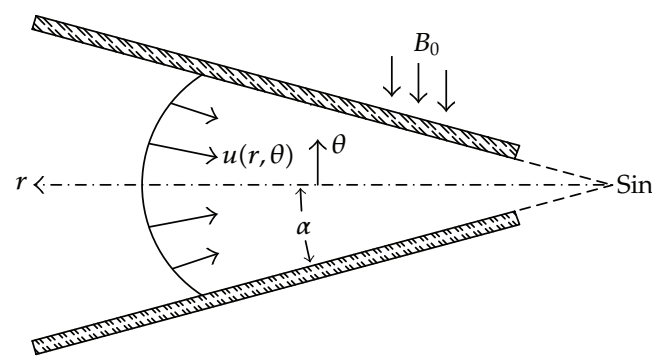

Inflow

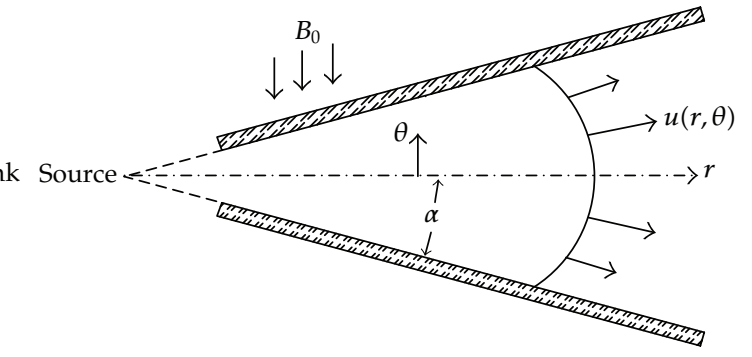

Outflow

Figure 1: The schematic diagram of MHD flow through convergent or divergent channels.

and there is no magnetic field along $z$-axis. The reduced form of continuity, Navier-Stokes and Maxwell's equations in polar system are

$$
\begin{gathered}
\rho \frac{\partial}{r \partial r}(r u(r, \theta))=0 \\
u(r, \theta) \frac{\partial u(r, \theta)}{\partial r}=-\frac{1}{\rho} \frac{\partial P}{\partial r}+v\left[\frac{\partial^{2} u(r, \theta)}{\partial r^{2}}+\frac{1}{r} \frac{\partial u(r, \theta)}{\partial r}+\frac{1}{r^{2}} \frac{\partial^{2} u(r, \theta)}{\partial \theta^{2}}-\frac{u(r, \theta)}{r^{2}}\right]-\frac{\sigma B_{0}^{2}}{\rho r^{2}} u(r, \theta) \\
\frac{1}{\rho r} \frac{\partial P}{\partial \theta}-\frac{2 v}{r^{2}} \frac{\partial u(r, \theta)}{\partial \theta}=0
\end{gathered}
$$

where $B_{0}$ is the electromagnetic induction strength, $\sigma$ the conductivity of the fluid, $u$ the velocity along radial direction, $P$ the fluid pressure, $v$ the coefficient of kinematic viscosity, and $\rho$ the fluid density. From (2.1),

$$
f(\theta)=r u(r, \theta) .
$$

Using dimensionless parameters,

$$
f(\eta)=\frac{f(\theta)}{f_{\max }}, \quad \eta=\frac{\theta}{\alpha}
$$

where $\alpha$ is the semiangle between the two inclined walls. Substituting (2.5) into (2.2) and (2.3) and eliminating $P$, one can obtain an ordinary differential equation for the normalized function profile $f(\eta)[3]$ :

$$
f^{\prime \prime \prime}(\eta)+2 \alpha \operatorname{Re} f(\eta) f^{\prime}(\eta)+(4-H) \alpha^{2} f^{\prime}(\eta)=0,
$$


where the Reynolds number is

$$
\operatorname{Re}=\frac{f_{\max } \alpha}{v}=\frac{U_{\max } r \alpha}{v} \begin{cases}\text { divergent-channel: } \alpha>0, & f_{\max }>0, \\ \text { convergent-channel: } \alpha<0, & f_{\max }<0 .\end{cases}
$$

Then we have boundary condition as follows:

$$
f(0)=1, \quad f^{\prime}(0)=0, \quad f(1)=0 .
$$

The Hartmann number is

$$
H=\sqrt{\frac{\sigma B_{0}^{2}}{\rho \mathcal{v}}}
$$

\section{Analytical Approximations by Means of DTM}

The differential transformation method is an analytical method for a vast variety of differential equations including ODEs and PDEs [15]. This method uses polynomials form to approximate the exact solutions. We now take a brief review to the DTM. The differential transform of the $k$ th derivative of function $f(t)$ is defined as follows [24]:

$$
F(k)=\frac{1}{k !}\left[\frac{d^{k} f(t)}{d t^{k}}\right]_{t=t_{0}},
$$

where $f(t)$ is the base function and $F(k)$ is the transformed function. The differential inverse transform of $F(k)$ is defined as

$$
f(t)=\sum_{k=0}^{\infty} F(k)\left(t-t_{0}\right)^{k}
$$

Equations (3.1) and (3.2) give the following:

$$
f(t)=\sum_{k=0}^{\infty} \frac{\left(t-t_{0}\right)^{k}}{k !}\left[\frac{d^{k} f(t)}{d t^{k}}\right]_{t=t_{0}} .
$$

This shows that differential transform is derived from Taylor series expansion, but the method does not evaluate the derivatives symbolically. However, relative derivatives are calculated by an iterative way which is described by the transformed equations of the base function. We approximate $f(t)$ by a finite series and (3.2) can be written as,

$$
f(t) \approx \sum_{k=0}^{N} F(k)\left(t-t_{0}\right)^{k}
$$


The main steps of the DTM are the following. First, we apply the differential transform (3.1) to the given differential equation or a system of differential equations to obtain a recursive relation. Second, solving the recursive relation and then using the differential inverse transform (3.2) we obtain the solution of the problem.

Using (3.1) and (3.2) the following theorems can be deduced.

Theorem 3.1. If $u(t)=x(t) \pm y(t)$, then $U(k)=X(k) \pm Y(k)$.

Theorem 3.2. If $u(t)=\alpha X(t)$, then $U(k)=\alpha X(k)$, where $\alpha$ is a constant.

Theorem 3.3. If $u(t)=\left(d^{m} x(t) / d t^{m}\right)$, then $U(k)=((m+k) ! / k !) X(k+m)$.

Theorem 3.4. If $u(t)=x(t) y(t)$, then $U(k)=\sum_{r}^{k} X(r) Y(k-r)$

Theorem 3.5. If $u(t)=t^{n}$, then $U(k)=\delta(k-n), \delta(k-n)= \begin{cases}1, & k=n, \\ 0, & k \neq n .\end{cases}$

Taking differential transform from (2.6), one can obtain:

$$
(k+1)(k+2)(k+3) F(k+3)+2 \alpha \operatorname{Re} \sum_{r=0}^{\infty}(r+1) F(r+1) F(k-r)+(4-H) \alpha^{2}(k+1) F(k+1)=0 .
$$

The boundary conditions, (2.8), are transformed into:

$$
F(0)=1, \quad F(1)=0, \quad \sum_{k=0}^{\infty} F(k)=0 .
$$

\section{Numerical Simulation}

Many categories of numerical methods have been developed for the numerical solution of differential equations; among them are the well-known fitted Runge-Kutta (RK) and RungeKutta-Nyström (RKN) methods [24]. In the last decade exponentially fitted RK and RKN methods have been constructed by many authors. Simos $[25,26]$ and Vanden Berghe et al. $[27,28]$ first constructed exponentially fitted RK methods. Also exponentially fitted RKN methods have been studied by Simos [29], Van de Vyver [30], and Franco [31].

In recent years, modern numerical techniques have been successfully employed to solve various types of mathematics and engineering problems. For example, Anastassi and Simos have applied Phase-fitted Runge-Kutta-Nystrom method for the numerical solution of initial value problems with oscillating solutions [32]. Simos has analyzed the Schrodinger equation by a new Numerov-type method [33]. The solution of the Schrodinger equation and related problems by means of the optimized explicit Runge-Kutta method with increased phase-lag order has been presented by Kosti et al. [34]. Stavroyiannis investigated the linear periodic initial value problems by nonlinear explicit two-step fourth-algebraic-order method [35]. The symmetric eight-step predictor-corrector method was applied for the radial Schrodinger equation and related IVPs with oscillating solutions by Panopoulos et al. [36]. Briefly speaking, this shows the great potential of modern numerical techniques for solving different problems in science and engineering. 
The shooting method works by considering the boundary conditions as a multivariable function of initial conditions at some point, reducing the boundary value problem to finding the initial conditions that give a root. The advantage of the shooting method is that it takes advantage of the speed and adaptivity of methods for initial value problems. The basic concept of the shooting method can be obtained from [37]. In this paper the shooting method, coupled with fourth-order Runge-Kutta scheme, is used for solving the problem of MHD flow through convergent or divergent channel in presence of a high magnetic field. An important point to note is that the numerical results have been obtained using Mathematica software. It is worth to mentioning that authors intend to apply some modern numerical techniques for the present problem and to compare the efficiency of different methods in the future.

\section{Results and Discussions}

The objective of the present study was to apply the differential transform method to obtain an explicit analytic solution of MHD flow through convergent or divergent channels in presence of a high magnetic field. The magnetic field plays its role in nondimensional parameter, namely, the Hartmann number. Figure 2 that shows velocity profile variation for a convergent channel with fixed Re number reveals the fact that by increasing magnetic field the velocity profile becomes flat and thickness of boundary layer decreases. In fact magnetic field induces a force in opposite of the momentum's direction that stabilizes the velocity profile. There is a reverse behavior in velocity profile as shown in Figure 3 for divergent channel, where we see that, by decreasing Hartman number, the velocity profile becomes flat and thickness of boundary layer decreases. As demonstrated in Figure 4 for pure inflow regime (negative Re number) in divergent channel, when Hartmann number increases we have a little change in velocity profile. Comparison of Figures 3 and 5 shows moderate increases in the velocity with increasing Hartmann numbers at small angle $\alpha=2.5^{\circ}$ and differences between velocity profiles are more noticeable at greater angle $\alpha=5^{\circ}$. Figures 6 and 7 show the velocity profile in divergent and convergent channels, respectively. In Figure 6, we can see that as $\alpha$ increases the effect of walls on fluid flow decreases when we move away from them which lead to an increase of velocity, while there is a reverse behavior in velocity profile as shown in Figure 7, where the values of $\alpha$ decrease. Figures 8 and 9 illustrate the effect of Reynolds numbers on the fluid velocity for fixed Hartmann numbers. According to Figure 8, for inflow regime as Re number increases, in a particular distance from the wall, velocity decreases and effects of backflow phenomenon are revealed. There is a reverse behavior in Figure 9 where as Re number increases, value of local velocity becomes more close to value of centerline velocity. We can infer from Figures 8 and 9 for inflow regime, back flow is prevented in the case of convergent channels but is possible for large Reynolds numbers in the case of divergent channels. Figures 8 and 9 show that there is a reverse condition for outflow regime.

For comparison, a few limited cases of the DTM solutions are compared with the numerical results. The comparison between the numerical results and DTM solution for velocity when $\operatorname{Re}=100$ and $H=1500$ is shown in Table 1 . The error bar shows an acceptable agreement between the results observed, which confirms the validity of the DTM. In this table error is introduced as Error $=\left|f(\eta)_{\mathrm{NM}}-f(\eta)_{\mathrm{DTM}}\right|$.

\section{Final Remarks}

In this investigation, the flow of an incompressible electricallyconducting viscous fluid in convergent or divergent channels under the influence of an externally applied homogeneous 


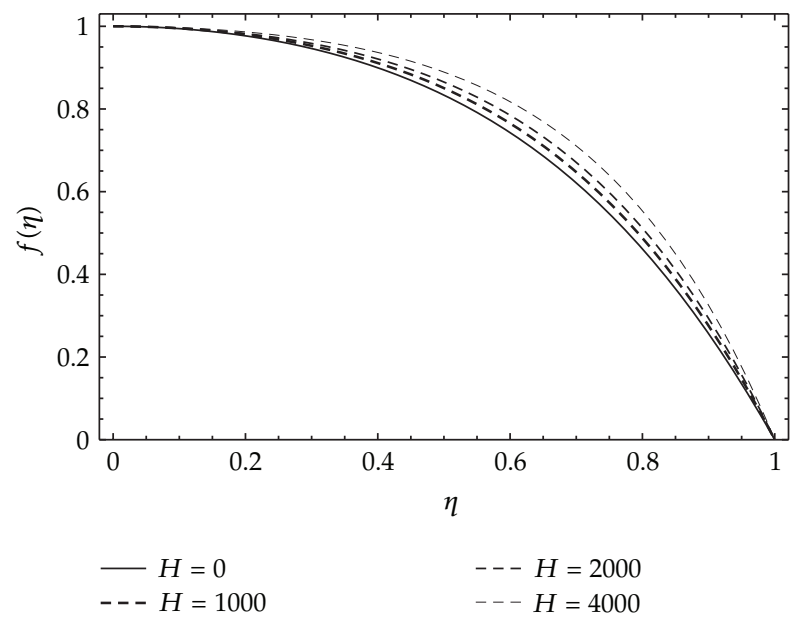

Figure 2: DTM solution for velocity in convergent channel for $\operatorname{Re}=100$ and $\alpha=-2.5^{\circ}$.

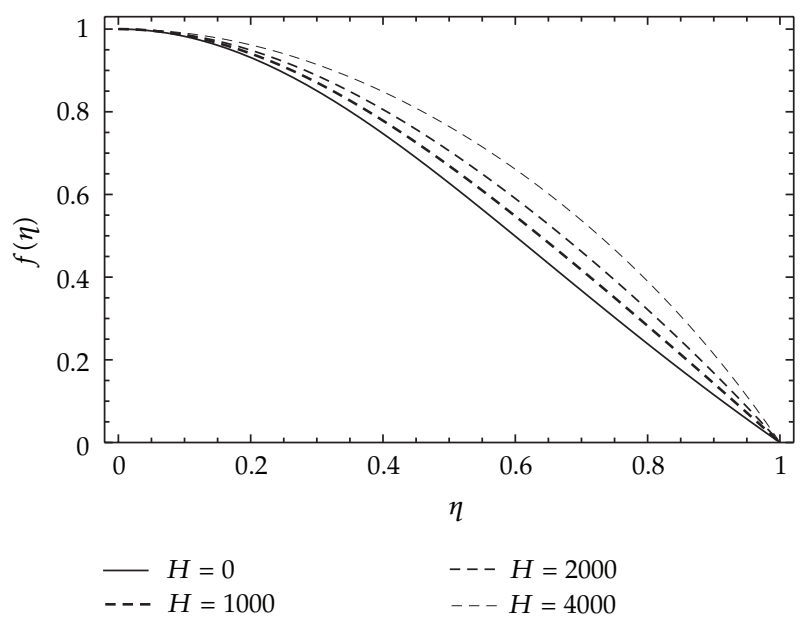

Figure 3: DTM solution for velocity in divergent channel for $\operatorname{Re}=100$ and $\alpha=2.5^{\circ}$.

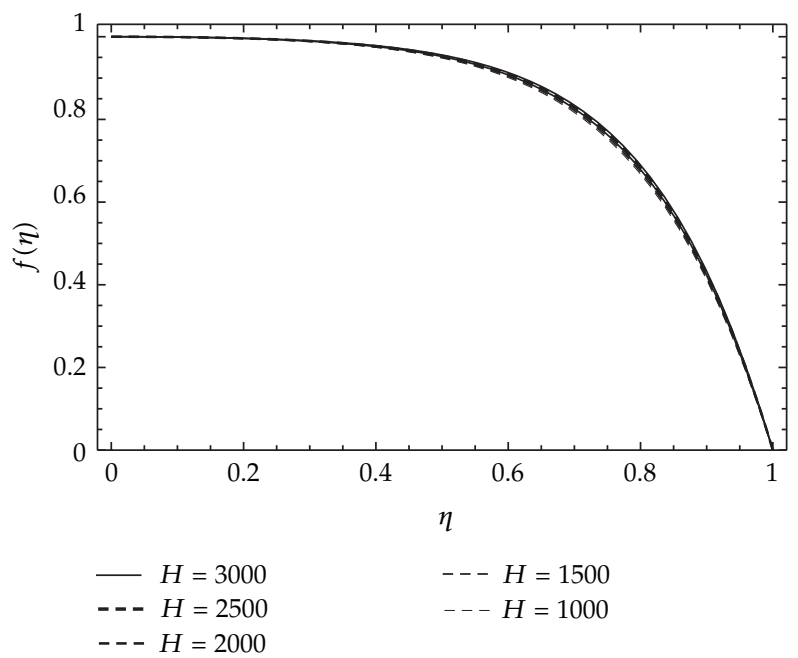

Figure 4: DTM solution for velocity in divergent channel for $\operatorname{Re}=-400$ and $\alpha=-2.5^{\circ}$. 


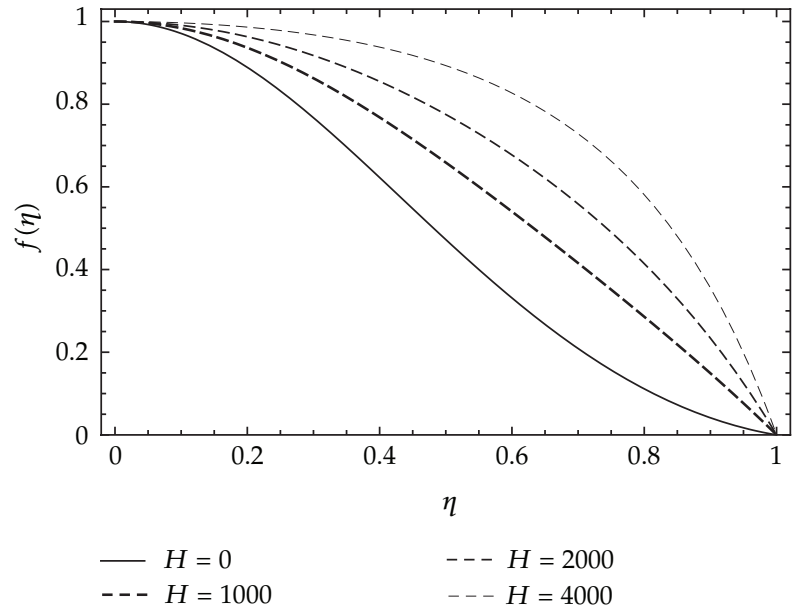

Figure 5: DTM solution for velocity in divergent channel for $\operatorname{Re}=100$ and $\alpha=5^{\circ}$.

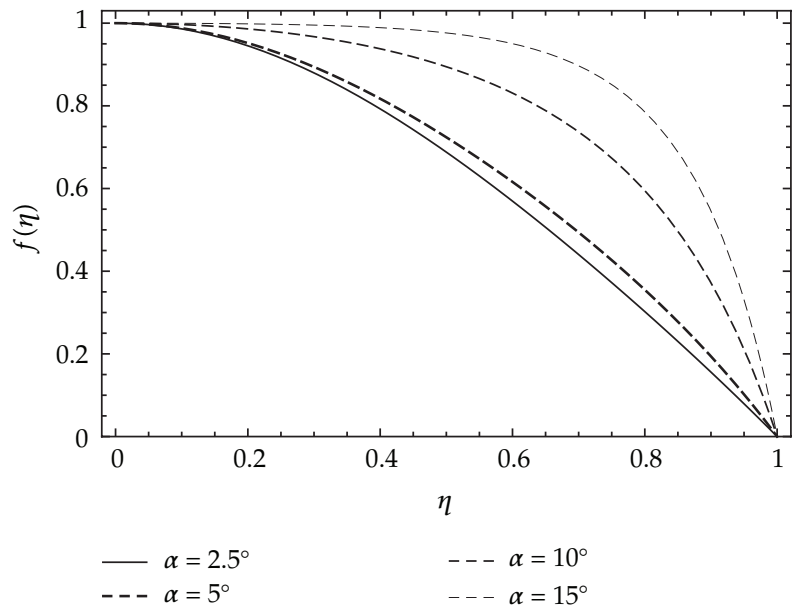

Figure 6: DTM solution for velocity in divergent channels for $\operatorname{Re}=100$ and $H=1500$.

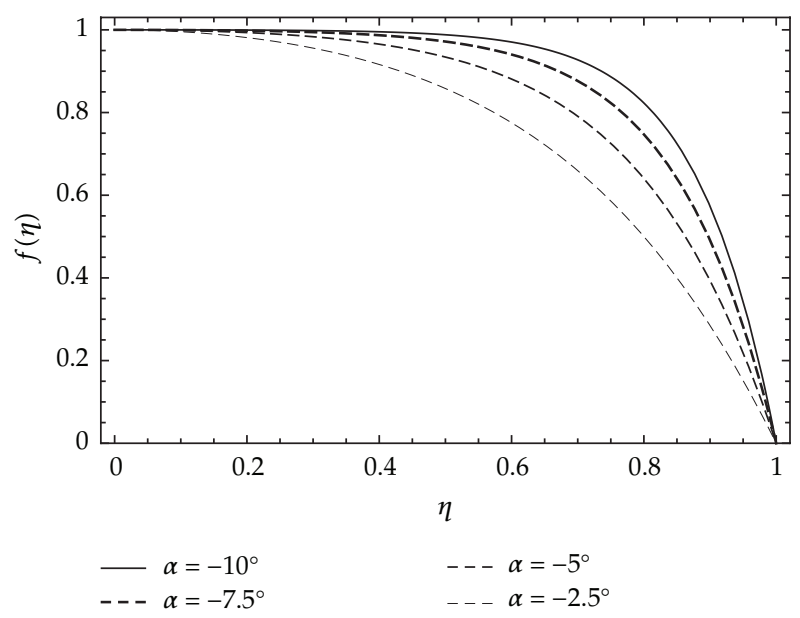

Figure 7: DTM solution for velocity in convergent channels for $\operatorname{Re}=100$ and $H=1500$. 


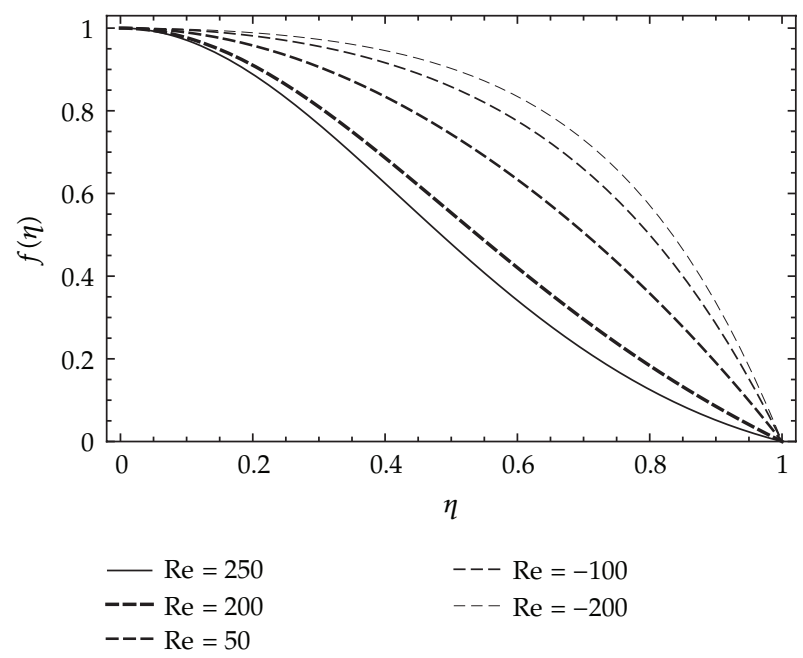

Figure 8: DTM solution for velocity in divergent channel for $\alpha=2.5^{\circ}$ and $H=1500$.

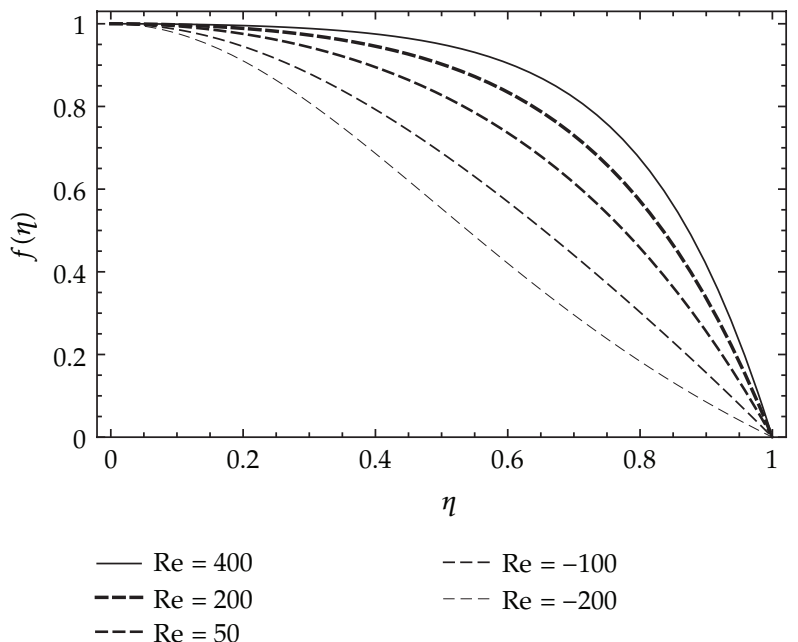

Figure 9: DTM solution for velocity in convergent channel for $\alpha=-2.5^{\circ}$ and $H=1500$.

magnetic field is studied both analytically and numerically. The governing highly nonlinear equation has been solved analytically using the differential transform method (DTM) and numerically by shooting method, coupled with fourth-order Runge-Kutta scheme. The behavior of the DTM solution is in good agreement with the numerical simulation. Graphical results are presented to investigate the influence of the angles of the channel, Hartmann number and Reynolds number on the velocity profiles. From the presented analysis, the following observations are noted.

(i) For a convergent channel with fixed Re number, with increasing the magnetic field, the velocity profile becomes flat and thickness of boundary layer decreases. While there is a reverse behavior in velocity profile for divergent channel. 
Table 1: The comparison between the numerical results and DTM solution for velocity when $\operatorname{Re}=100$ and $H=1500$.

\begin{tabular}{|c|c|c|c|c|c|c|}
\hline \multirow{2}{*}{$\eta$} & \multicolumn{3}{|c|}{$\alpha=2.5^{\circ}$} & \multicolumn{3}{|c|}{$\alpha=-2.5^{\circ}$} \\
\hline & DTM & Numerical & Error & DTM & Numerical & Error \\
\hline 0.00 & 1.00000000 & 1.00000000 & 0.00000000 & 1.00000000 & 1.00000000 & 0.00000000 \\
\hline 0.05 & 0.99651592 & 0.99651592 & 0.00000000 & 0.99887174 & 0.99887174 & 0.00000000 \\
\hline 0.10 & 0.98611456 & 0.98611455 & 0.00000001 & 0.99545428 & 0.99545428 & 0.00000000 \\
\hline 0.15 & 0.96894623 & 0.96894623 & 0.00000000 & 0.98964873 & 0.98964873 & 0.00000000 \\
\hline 0.20 & 0.94525393 & 0.94525392 & 0.00000001 & 0.98128773 & 0.98128772 & 0.00000001 \\
\hline 0.25 & 0.91536246 & 0.91536246 & 0.00000000 & 0.97013152 & 0.97013151 & 0.00000001 \\
\hline 0.30 & 0.87966426 & 0.87966426 & 0.00000000 & 0.95586263 & 0.95586262 & 0.00000001 \\
\hline 0.35 & 0.83860260 & 0.83860260 & 0.00000000 & 0.93807899 & 0.93807898 & 0.00000001 \\
\hline 0.40 & 0.79265312 & 0.79265312 & 0.00000000 & 0.91628580 & 0.91628578 & 0.00000002 \\
\hline 0.45 & 0.74230468 & 0.74230468 & 0.00000000 & 0.88988619 & 0.88988617 & 0.00000002 \\
\hline 0.50 & 0.68804028 & 0.68804027 & 0.00000001 & 0.85817118 & 0.85817117 & 0.00000001 \\
\hline 0.55 & 0.63031878 & 0.63031877 & 0.00000001 & 0.82030940 & 0.82030938 & 0.00000002 \\
\hline 0.60 & 0.56955800 & 0.56955800 & 0.00000000 & 0.77533733 & 0.77533732 & 0.00000001 \\
\hline 0.65 & 0.50611946 & 0.50611946 & 0.00000000 & 0.72215163 & 0.72215162 & 0.00000001 \\
\hline 0.70 & 0.44029483 & 0.44029483 & 0.00000000 & 0.65950492 & 0.65950492 & 0.00000000 \\
\hline 0.75 & 0.37229410 & 0.37229410 & 0.00000000 & 0.58600786 & 0.58600786 & 0.00000000 \\
\hline 0.80 & 0.30223504 & 0.30223503 & 0.00000001 & 0.50014060 & 0.50014060 & 0.00000000 \\
\hline 0.85 & 0.23013349 & 0.23013349 & 0.00000000 & 0.40027797 & 0.40027799 & 0.00000002 \\
\hline 0.90 & 0.15589396 & 0.15589396 & 0.00000000 & 0.28473379 & 0.28473382 & 0.00000003 \\
\hline 0.95 & 0.07929949 & 0.07929949 & 0.00000000 & 0.15183034 & 0.15183037 & 0.00000003 \\
\hline 1.00 & 0.00000000 & 0.00000000 & 0.00000000 & 0.00000000 & 0.00000004 & 0.00000004 \\
\hline
\end{tabular}

(ii) For a divergent channel, increasing in the Reynolds numbers leads to adverse pressure gradient causing velocity reduction near the walls.

(iii) For pure outflow regime (positive Re number), there are moderate increases in the velocity with increasing Hartmann number.

(iv) For divergent channels, as $\alpha$ increases the effect of walls on fluid flow decreases when we move away from them which lead to an increase of velocity, while there is a reverse behavior in velocity profile for convergent channels.

(v) For inflow regime (negative Re number), as Re number increases, in a particular distance from the wall, velocity decreases and effects of backflow phenomenon are revealed.

\section{References}

[1] G. B. Jeffery, "The two-dimensional steady motion of a viscous fluid," Philosophical Magazine, vol. 6, pp. 455-465, 1915.

[2] G. Hamel, "Spiralförmige Bewgungen Zäher Flüssigkeiten," Jahresber Deutsch Math-Verein, vol. 25, pp. 34-60, 1916.

[3] H. Schlichting, Boundary Layer Theory, McGraw-Hill, New York, NY, USA, 1955.

[4] L. Rosenhead, "The steady two-dimensional radial flow of viscous fluid between two inclined plane walls," Proceedings of the Royal Society A, vol. 175, pp. 436-467, 1940. 
[5] K. Batchelor, An Introduction to Fluid Dynamics, Cambridge University Press, 1967.

[6] I. J. Sobey and P. G. Drazin, "Bifurcations of two-dimensional channel flows," Journal of Fluid Mechanics, vol. 171, pp. 263-287, 1986.

[7] L. E. Fraenkel, "Laminar flow in symmetrical channels with slightly curved walls. I. On the JefferyHamel solutions for flow between plane walls," Proceedings of the Royal Society A, vol. 267, pp. 119-138, 1962.

[8] O. D. Makinde and P. Y. Mhone, "Hermite-Padé approximation approach to MHD Jeffery-Hamel flows," Applied Mathematics and Computation, vol. 181, no. 2, pp. 966-972, 2006.

[9] M. Hamadiche, J. Scott, and D. Jeandel, "Temporal stability of Jeffery-Hamel flow," Journal of Fluid Mechanics, vol. 268, pp. 71-88, 1994.

[10] A. McAlpine and P. G. Drazin, "On the spatio-temporal development of small perturbations of JefferyHamel flows," Fluid Dynamics Research, vol. 22, no. 3, pp. 123-138, 1998.

[11] S. M. Moghimi, G. Domairry, S. Soleimani, E. Ghasemi, and H. Bararnia, "Application of homotopy analysis method to solve MHD Jeffery-Hamel flows in non-parallel walls," Advances in Engineering Software, vol. 42, no. 3, pp. 108-113, 2011.

[12] R. Nijsing and W. Eifler, "A computational analysis of transient heat transfer in fuel rod bundles with single phase liquid metal cooling," Nuclear Engineering and Design, vol. 62, no. 1-3, pp. 39-68, 1980.

[13] L. Bansal, Magnetofluiddynamics of Viscous Fluids, Jaipur Publishing House, Jaipur, India, 1994.

[14] R. Moreau, Magnetohydrodynamics, vol. 3 of Fluid Mechanics and its Applications, Kluwer Academic Publishers, Dordrecht, The Netherlands, 1990.

[15] J. K. Zhou, Differential Transformation and Its Applications for Electrical Circuits, Huazhong University Press, Wuhan, China, 1986.

[16] J. Biazar and M. Eslami, "Analytic solution for Telegraph equation by differential transform method," Physics Letters. A, vol. 374, no. 29, pp. 2904-2906, 2010.

[17] M. S. Abel, N. Mahesha, and J. Tawade, "Heat transfer in a liquid film over an unsteady stretching surface with viscous dissipation in presence of external magnetic field," Applied Mathematical Modelling, vol. 33, no. 8, pp. 3430-3441, 2009.

[18] F. Ayaz, "Solutions of the system of differential equations by differential transform method," Applied Mathematics and Computation, vol. 147, no. 2, pp. 547-567, 2004.

[19] A. Arikoglu and I. Ozkol, "Solution of boundary value problems for integro-differential equations by using differential transform method," Applied Mathematics and Computation, vol. 168, no. 2, pp. 1145-1158, 2005.

[20] H. Liu and Y. Song, "Differential transform method applied to high index differential-algebraic equations," Applied Mathematics and Computation, vol. 184, no. 2, pp. 748-753, 2007.

[21] A. S. V. Ravi Kanth and K. Aruna, "Differential transform method for solving the linear and nonlinear Klein-Gordon equation," Computer Physics Communications, vol. 180, no. 5, pp. 708-711, 2009.

[22] Z. M. Odibat, C. Bertelle, M. A. Aziz-Alaoui, and G. H. E. Duchamp, "A multi-step differential transform method and application to non-chaotic or chaotic systems," Computers $\mathcal{E}$ Mathematics with Applications, vol. 59, no. 4, pp. 1462-1472, 2010.

[23] R. K. Rathy, An Introduction to Fluid Dynamics, Oxford \& IBH Publishing Co., New Delhi, India, 1976.

[24] J. R. Cash and S. Girdlestone, "Variable step Runge-Kutta-Nyström methods for the numerical solution of reversible systems," Journal of Numerical Analysis, Industrial and Applied Mathematics, vol. 1, no. 1, pp. 59-80, 2006.

[25] T. E. Simos, "An exponentially-fitted Runge-Kutta method for the numerical integration of initialvalue problems with periodic or oscillating solutions," Computer Physics Communications., vol. 115, no. 1 , pp. 1-8, 1998.

[26] T. E. Simos, "Exponentially fitted Runge-Kutta methods for the numerical solution of the Schrödinger equation and related problems," Computational Materials Science, vol. 18, no. 3-4, pp. 315-332, 2000.

[27] L. Gr. Ixaru and G. Vanden Berghe, Exponential Fitting, vol. 568 of Mathematics and Is Applications, Kluwer Academic Publishers, Dordrecht, The Netherlands, 2004.

[28] G. Vanden Berghe, H. De Meyer, M. Van Daele, and T. Van Hecke, "Exponentially-fitted explicit Runge-Kutta methods," Computer Physics Communications, vol. 123, no. 1-3, pp. 7-15, 1999.

[29] T. E. Simos, "Exponentially-fitted Runge-Kutta-Nyström method for the numerical solution of initialvalue problems with oscillating solutions," Applied Mathematics Letters, vol. 15, no. 2, pp. 217-225, 2002.

[30] H. Van De Vyver, "A symplectic exponentially fitted modified Runge-Kutta-Nyström method for the numerical integration of orbital problems," New Astronomy, vol. 10, no. 4, pp. 261-269, 2005. 
[31] J. M. Franco, "Exponentially fitted explicit Runge-Kutta-Nyström methods," Journal of Computational and Applied Mathematics, vol. 167, no. 1, pp. 1-19, 2004.

[32] D. F. Papadopoulos, Z. A. Anastassi, and T. E. Simos, "A phase-fitted Runge-Kutta-Nyström method for the numerical solution of initial value problems with oscillating solutions," Computer Physics Communications, vol. 180, no. 10, pp. 1839-1846, 2009.

[33] T. E. Simos, "A new Numerov-type method for the numerical solution of the Schrödinger equation," Journal of Mathematical Chemistry, vol. 46, no. 3, pp. 981-1007, 2009.

[34] A. A. Kosti, Z. A. Anastassi, and T. E. Simos, "An optimized explicit Runge-Kutta method with increased phase-lag order for the numerical solution of the Schrödinger equation and related problems," Journal of Mathematical Chemistry, vol. 47, no. 1, pp. 315-330, 2010.

[35] S. Stavroyiannis and T. E. Simos, "A nonlinear explicit two-step fourth algebraic order method of order infinity for linear periodic initial value problems," Computer Physics Communications, vol. 181, no. 8, pp. 1362-1368, 2010.

[36] G. A. Panopoulos, Z. A. Anastassi, and T. E. Simos, “A symmetric eight-step predictor-corrector method for the numerical solution of the radial Schrödinger equation and related IVPs with oscillating solutions," Computer Physics Communications, vol. 182, no. 8, pp. 1626-1637, 2011.

[37] S. M. Roberts and J. S. Shipman, Two-Point Boundary Value Problems: Shooting Methods, Modern Analytic and Computational Methods in Science and Mathematics, No. 3, American Elsevier, New York, NY, USA, 1972. 


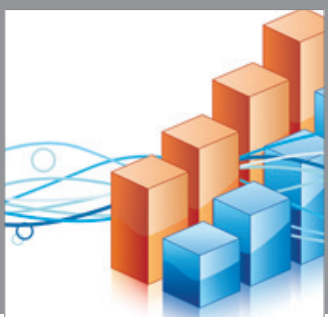

Advances in

Operations Research

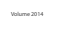

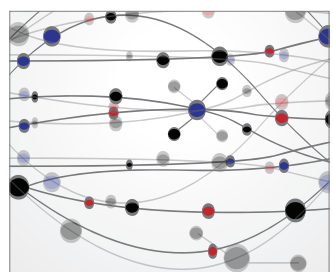

\section{The Scientific} World Journal
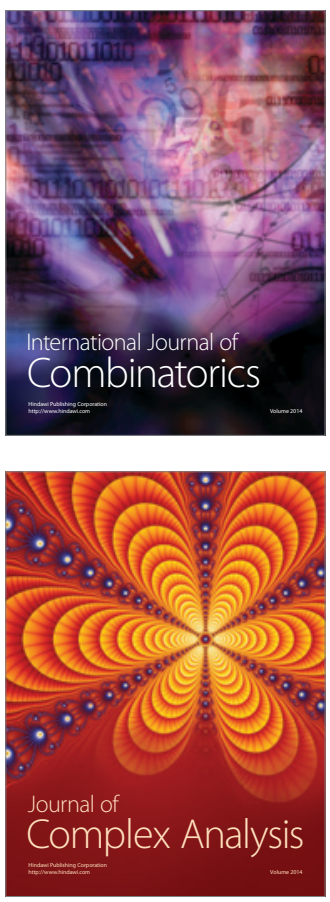

International Journal of

Mathematics and

Mathematical

Sciences
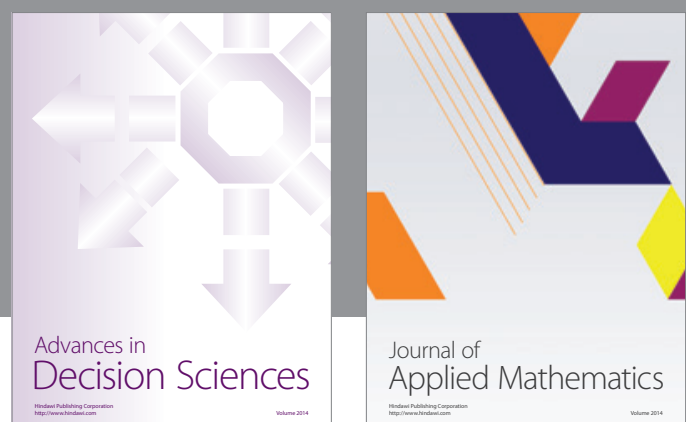

Journal of

Applied Mathematics
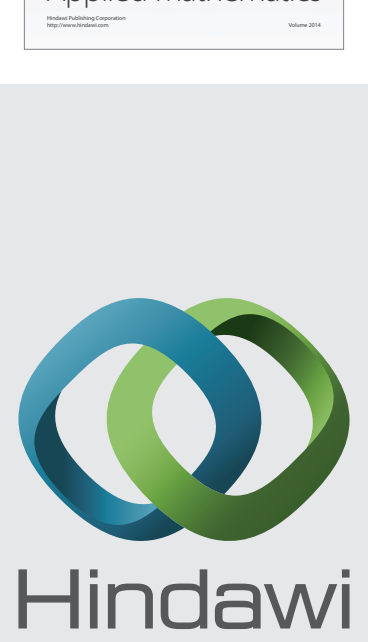

Submit your manuscripts at http://www.hindawi.com
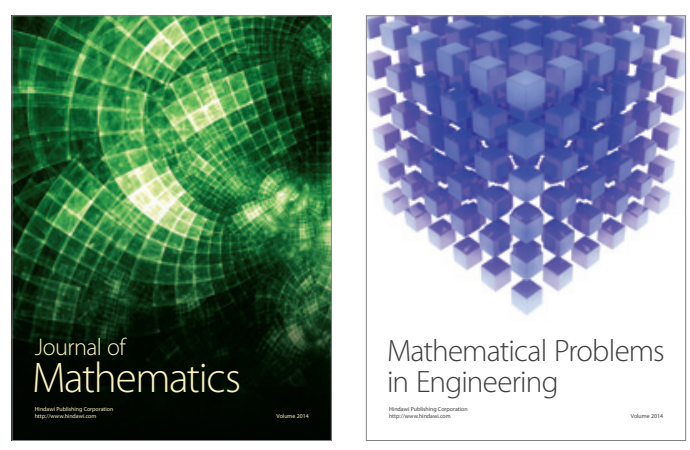

Mathematical Problems in Engineering
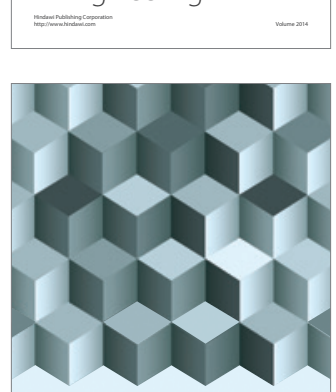

Journal of

Function Spaces
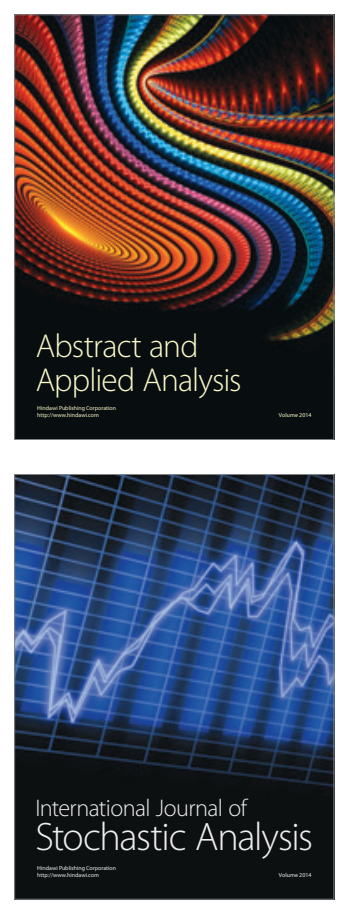

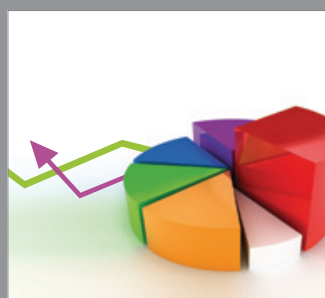

ournal of

Probability and Statistics

Promensencen
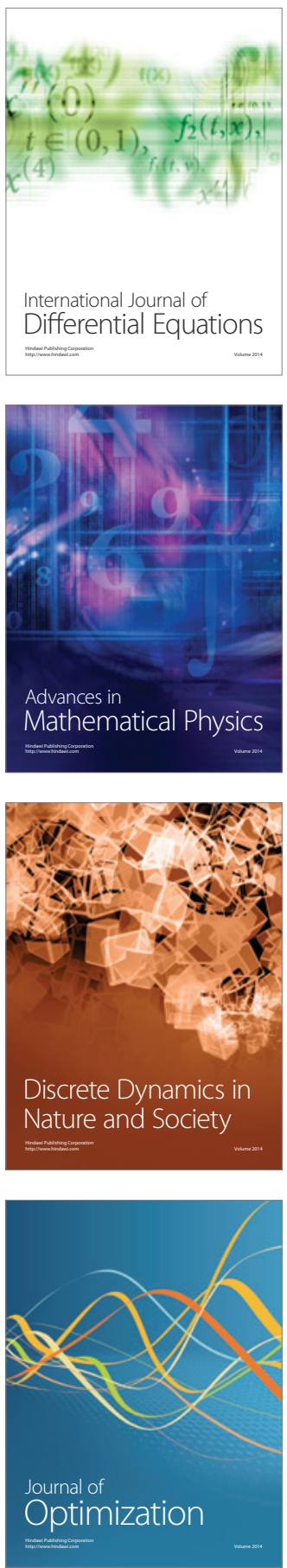